\title{
SARS-CoV-2 airborne transmission: a validated sampling and analytical method
}

Angelo Robotto $^{1}$, Andrea Civra ${ }^{2 \#}$, Paola Quaglino ${ }^{1}$, Denis Polato $^{1}$, Enrico Brizio ${ }^{1}$, David Lembo $^{2}$

${ }^{1}$ Environmental Protection Agency of Piedmont (Arpa Piemonte), Via Pio VII 9, 10135, Torino, Italy.

${ }^{2}$ Dept. of Clinical and Biological Science, University of Torino, Regione Gonzole 10, 10043, Orbassano, Torino, Italy.

\# Corresponding Authors: Andrea Civra

Dept. of Clinical and Biological Science, University of Torino,

Regione Gonzole 10,

10043, Orbassano, Torino, Italy

Tel.: +39-0116705485; Fax: +39-0112365484

E-mail: andrea.civra@unito.it 


\begin{abstract}
The most recent scientific studies have finally identified the airborne transmission of SARS-CoV-2 as significant. Therefore, the airborne transmission path for SARS-CoV-2 is of primary scientific and health-related interest as it could actually involve management, accessibility, use and functionality of many activities, including hospitals (where COVID wards represent only a part of the critical issues), schools, workplaces, offices, factories, means of transport, sports venues, and the outdoor environment. It is necessary to develop a sampling and analytical method for virus-laden bioaerosol that could be considered reliable and validated according to ISO/IEC 17025 requirements.

The present paper defines samples pretreatments aiming at recover SARS-CoV-2 from glass-fiber and PTFE filters used by low and high-volume air samplers. Recovery test results focused on the sample concentration step carried out by means of ultracentrifugation are reported as well. Human coronavirus strain OC43 (a surrogate $\beta$-coronavirus with same SARS-CoV-2 particle structure) was used to validate each step of the recovery tests.

We obtained the following results:

- the recovery efficiency from glass-fiber filters and quartz filters could be strongly enhanced by using an elution buffer containing up to $40 \%$ of fetal calf serum.

- the recovery efficiency of coronavirus OC43 (HCoV-OC43) from PTFE filters is much higher and easier than from glass-fiber filters; for glass-fiber filters, we found that a two-step procedure is necessary to elute viral infective particles: a 3 hour-shaking step, followed by a 30 seconds-vortexing step. For PTFE 60 minutes-shaking is enough.

- the effect of suction time on filters could be resumed as follows: concerning $10 \mathrm{~cm}$ glass-fiber filters, sampling durations up to 20 minutes at a flow rate of $500 \mathrm{~L}$ per minute do not affect recovery efficiencies. On the contrary, the recovery efficiency of infectious virions from $4.7 \mathrm{~cm}$ PTFE filters decreases of a factor 2 after 3 hours of sampling at a flow rate of $20 \mathrm{~L}$ per minute.

- the recovery efficiency of ultracentrifugation is $57 \%$.
\end{abstract}

Furthermore, the effect of the storage temperature of the filters immersed in the transport medium on the infectivity of HCoV-OC43 has been assessed.

Based on the sampling techniques and the analytical methods developed as described in the present study, many field tests were carried out reporting virus concentrations up to 50 genomic copies per cubic meter of air in domestic environment with poor ventilation condition, whereas in hospital wards 
the detectable concentrations of SARS-CoV-2 were generally lower than 10 genomic copies per cubic meter of air.

The developed methods, aiming at providing the community with reliable determinations about the presence of SARS-CoV-2 and other airborne pathogens in air, prove essential for the development, during the pandemic, of a coherent management of places (especially of crowded ones) such as means of transport, stations, gyms, theaters, cinemas.

Keywords:

SARS-CoV-2

Airborne transmission

PTFE filter

Glass-fiber filter

Bioaerosol

High-volume sampler 


\section{Introduction}

SARS-CoV-2 is a pathogenic virus belonging to the Coronaviridae family. The infection causes a syndrome called COVID-19 that, in the most severe cases, is characterized by pneumonia and loss of respiratory function. Differently from other member of Coronaviridae family that can cause severe pathology in the lower respiratory airways (namely SARS-CoV and MERS-CoV), SARS-CoV-2 apparently adapted better to the human host (Platto S et al., 2020), being characterized by a high transmissibility that led to a pandemic spread. On 30 January 2020, the World Health Organization (WHO) declared SARS-CoV-2 outbreak as a public health emergency of international concern (PHEIC), WHO's highest level of alarm.

SARS-CoV-2 infection is transmitted by different routes, namely (1) direct or indirect contact with respiratory secretions - emitted from cough, sneeze and even normal speaking by positive people, even if they are asymptomatic (Morawska and Cao, 2020), or (2) airborne transmission, defined as the spread of an infectious agent caused by the dissemination of aerosols that remain infectious when suspended in air over long distances and time (WHO, 2020c). These small particles of pathogencontaining respiratory secretions expelled into the air can remain airborne for long periods (Atkinson et al., 2009), carrying their contents away from where they were originated (Siegel et al., 2007), therefore raising the possibility of transmission also in the absence of aerosol-generating procedures. Notably, on April 5th, 2021, the ASHRAE Epidemic Task Force released an updated statement on the airborne transmission of SARS-CoV-2, acknowledging its risk in indoor environment: "Airborne transmission of SARS-CoV-2 is significant and should be controlled. Changes to building operations, including the operation of heating, ventilating, and air-conditioning systems, can reduce airborne exposures" (ASHRAE, 2021) This statement replaces the April 2020 one (ASHRAE, 2020), that referred to airborne transmission as "sufficiently likely". On the contrary, again on April $5^{\text {th }}, 2021$, the Center for Disease Control and Prevention of the USA reports that "the risk of SARS-CoV-2 infection via the fomite transmission route is low, and generally less than 1 in 10,000, which means that each contact with a contaminated surface has less than a 1 in 10,000 chance of causing an infection" (CDC, 2021).

Although close contact with contagious people is generally considered to be the main way the pandemic spreads, several obstacles prevent from clarifying completely the actual epidemiological role played by airborne transmission. Firstly, most part of the studies that address this topic are focused only on the detection of viral genome in the air, and do not correlate these molecular biology data with a solid assessment of infective viral particles. While indirect and analogy-based evidences have been provided by comparing the infectivity of SARS-CoV and MERS-CoV in air with the one 
of SARS-CoV-2 particles (da Silva PG et al., 2021), to date only few published studies have provided information on SARS-CoV-2 viability in air, in particular in hospital wards dedicated to COVID-19 patients (Binder et al., 2020; Lednicky et al., 2020; Santarpia et al., 2020; van Doremalen et al., 2020). Interestingly, the findings of these studies provided useful hints about SARS-CoV-2 spread, supporting the position that aerosol transmission can occur early on in the course of disease, before COVID-19 patients are ill enough to require hospitalization, concluding that aerosol transmission should be assessed soon after symptoms onset (Binder et al., 2020).

The lack of more studies aiming at monitoring the presence of SARS-CoV-2 infective particles in the air represents a remarkable knowledge gap that requires urgent attention, and is ascribable to the technical limits that hinder the development of reliable techniques to capture and isolate infective particles in the air: in other words, in order to perform an adequate monitoring of the presence of SARS-CoV-2 in indoor and outdoor air, it is necessary to develop a bioaerosol sampling method capable of maintaining the infectivity of the viral particles.

Various sampling devices can be used to capture bioaerosol containing viruses (Verrault et al., 2008; Pan et al., 2019). The most common ones are solid impactors, liquid impactors and filters, whose sampling technology is based on a few principles such as inertia, Brownian motions, adhesion properties, mainly depending on the aerodynamic diameter of the airborne particles.

Solid impactors, such as Andersen samplers, slit samplers and cyclone samplers, are usually more efficient at capturing large particles. Despite recent advancements, cut-off sizes (particle diameter with $50 \%$ collection efficiency) for cyclons (mostly $>1 \mu \mathrm{m}$ ) cannot meet the sampling requirements for small sized virus-containing particles. Moreover, the cyclone action can damage and deactivate viruses, resulting in an underestimate of the infectious viruses collected.

Liquid impactors are liquid impingers using different liquid solutions such as sterile distilled water, physiological saline, phosphate-buffered saline, nutrient broth, peptone water, or mineral oil to collect particles. All-Glass Impingers (AGI) samplers, characterized by a critical flow orifice, accelerating the air passing through it to sonic velocity, are the most often used samplers for the capture of airborne viruses; the formation of air bubbles in the liquid phase can improve the collection of small particles through diffusion but part of the sample could be lost because of transport of droplets towards the extraction pump or particles re-aerosolization, thus limiting sampling durations. Nevertheless, collection through liquid media prevents desiccation and allows the preservation of the infectivity of the sample. A "swirling aerosol collector" with three tangential sonic nozzles causing a swirling motion of the liquid phase has been developed to make the sampling procedure less violent and destructive than with traditional AGI sampler. The tangential components of the aerosol jets produce 
swirling air motion inside the device that rotates the liquid and swirls it up the inner wall thus wetting the region where the aerosol jets meet the inner wall. This way, swirling aerosol collectors can improve collection efficiency of particles smaller than $1 \mu \mathrm{m}$ up to $80 \%$ at $0.3 \mu \mathrm{m}$, as highlighted by Willeke et al. (1998); moreover, this kind of sampler is suitable to high-viscosity non-evaporating collection fluid, such as white mineral oil, thus permitting longer sampling times, up to 8 hours (Lin et al., 1999).

On the other hand, filters are the most effective device to capture submicrometric particles; they could be made of cellulose, polycarbonate, glass-fibers, quartz fibers, polytetrafluoroethylene (PTFE). The above-cited literature reports that $0.3 \mu \mathrm{m}$ PTFE filters appear to be the best option for long-term sampling of 10 to $900 \mathrm{~nm}$ diameter virus-laden particles.

Filters are very efficient, but literature reports that they can cause desiccation of the sample, likely compromising virus infectivity (Verrault et al., 2008). Modern molecular biology techniques can detect both viral genome and antigens, so they do not require viral particle integrity to be maintained to detect viruses; however, prolonged filtration could also damage and reduce the amount of detectable genetic material, making its final concentration assessment less reliable. Moreover, in order to be further analyzed, the genetic material captured by filters must be eluted by a suitable solution.

A partial solution could be represented by gelatine filters, because they are very efficient while they do not appear to significantly affect viral infectivity: they can be dissolved into liquid for molecular or virus enumeration in cell cultures without significantly affecting the viability of many viruses. Some authors report that low humidity can cause them to dry and break, while high humidity can cause dissolution of the filter; therefore, the sampling duration is usually quite short, around 15 minutes (Pan et al., 2019). On the contrary, studies of some manufacturer demonstrated that gelatine membranes could be qualified for continuous air monitoring in industrial pharmaceutical production environments covering a whole $8 \mathrm{~h}$ work shift without the need for human intervention (Scherwing et al, 2019).

Among the described bioaerosol sampling technologies, the most suitable should be chosen to match the following minimal requirements, defined also in a previous study produced by our group (Robotto et al., 2021):

1. sampling large volumes of air in consideration of the expected dilution in environmental samples;

2. enhancement of bioaerosol capture efficiency, for fractions smaller than $1 \mu \mathrm{m}$;

3. preservation of sample infectivity, to allow viral replication in vitro on susceptible cell cultures; 
4. having temperatures not hostile to the pathogen throughout the sampling process and the sample transport and manipulation;

5. reduction of degradation or re-aerosolization, bounce, inlet and wall losses in samplers;

6. establishing standardized procedures and methods for sampling airborne viruses and enable measurement of the detection limit of the virus samplers;

7. evaluation and use of optimal media for suspension or collection;

8. optimization of sample pre-treatment (elution from filters, concentration of transport medium, RNA extraction).

Therefore, a conceptual approach like the one described by Robotto et al. (2021) should be developed to allow the choice of suitable collection materials, sampling duration, transport medium and sample pre-treatments with a reliable and reproducible plan. Recovery tests represent a necessary step to validate an analytical method, assuring the right quality to environmental sampling and analysis according to ISO/IEC 17025 standard.

To address these points, in the present study we provide evidence of the feasibility and reproducibility of the approaches described in our previous paper. 


\section{Materials and Methods}

\section{Sampling devices and filters}

In the last 10 months our research group formed by the Environmental Protection Agency of Piedmont (Arpa Piemonte) and Università degli Studi di Torino, Dept. of Clinical and Biological Science, Azienda Ospedaliero-Universitaria San Luigi Gonzaga, focused on the use of 3 different sampling devices:

1) a low-volume sampler for air filtration on PTFE filters, with a flow rate of $20 \mathrm{~L} / \mathrm{min}$;

2) a high-volume sampler for air filtration on glass-fiber or quartz filters, with a flow rate of 500 L/min;

3) a swirling aerosol collector (BioSampler, SKC Inc., Eighty-Four, PA, USA) accelerating the flow of aspirated air to sonic speed, minimizing losses due to evaporation, maintaining the infectivity and integrity of the viral particles by transferring them directly to a suitable transport solution (phosphate buffered saline (PBS), Dulbecco's Modified Eagle Medium (DMEM, Sigma, St. Louis, MO, USA) or non-aqueous fluids), sampling a flow rate of $12.5 \mathrm{~L} / \mathrm{min}$.

Concerning air filtration, we employed:

- $\quad$ PTFE filters $(0.2-\mu \mathrm{m}$ pore size, diameter of $4.7 \mathrm{~cm}$, Sartorius AG, Göttingen, Germany)

- $\quad$ glass-fiber filters (grade MG G, $1.5-\mu \mathrm{m}$ pore size, diameter of $4.7 \mathrm{~cm}$ and $10 \mathrm{~cm}$, Munktell Filter AB, Falun, Sweden)

\section{Cell lines and viruses}

Human lung fibroblast MRC-5 (ATCC® CCL-171) were propagated in DMEM (Sigma, St. Louis, MO, USA) supplemented with $1 \%$ (v/v) penicillin/streptomycin solution (Euroclone, Milan, Italy) and heat inactivated, $10 \%$ (v/v) fetal bovine serum (Sigma). Human coronavirus strain OC43 (HCoVOC43) (ATCC® VR-1558) was purchased from ATCC (American Type Culture Collection, Rockville, MD, USA) and propagated in MRC-5 cells, at $34^{\circ} \mathrm{C}$, in a humidified $5 \% \mathrm{CO} 2$ incubator. When the full cytopathic effect (CPE) developed, cells and supernatants were harvested, pooled, frozen, and thawed three times, then clarified and aliquoted. The virus was stored at $-80^{\circ} \mathrm{C} . \mathrm{HCoV}$ OC43 titers were determined by the indirect immunoperoxidase staining procedure. Briefly, MRC-5 cells were seeded 2 days before infection in 96-well plates, reaching 60\%-70\% confluence at the time of infection. The viral suspension was serially diluted in DMEM supplemented with $2 \%$ fetal bovine serum and inoculated; the infected wells were incubated at $34^{\circ} \mathrm{C}$ for 24 hours, allowing viruses to 
enter the cells and replicate. After this time, cells were washed with medium, and fixed with cold acetone-methanol (50:50). Cells were then permeabilized with Triton X-100 0.1\% in PBS, and incubated with an OC43-specific monoclonal antibody (MAB9013; Merck Life Science Srl, Milan, Italy). After three quick washes with PBS, the secondary antibody peroxidase-conjugated AffiniPure F(ab')2 Fragment Goat Anti-Mouse IgG (H + L) (Jackson ImmunoResearch Laboratories Inc., 872 W. Baltimore Pike, West Grove, PA 19390) was added. Finally, after three more washes with PBS, 3, 3' diaminobenzidine tetrahydrochloride (DAB Substrate; 11718096001, Merck Life Science Srl, Milan, Italy) was added. It is one of the major sensitive substrates for HRP (horseradish peroxidase): the reaction product is brown in color and insoluble, thereby precipitating over the antigen-antibody site and staining infected cells. Viral titers are expressed as focus-forming unit (FFU) per ml.

\section{Virus-filter elution procedures}

\section{Glass-fiber filters}

A fixed inoculum of $\mathrm{HCoV}-\mathrm{OC} 43$ suspension $(50 \mu \mathrm{l})$ was spotted on glass-fiber filters, alternatively of $4.7 \mathrm{~cm}$ or $10 \mathrm{~cm}$. Different procedures were tested, in order to develop a method to elute viral particles collected on the filter. In particular, three different approaches were explored, namely (A) a wash procedure, (B) a vortexing procedure, and (C) a shaking procedure.

For the wash procedure, $4 \mathrm{ml}$ of DMEM supplemented with $2 \%, 10 \%$, or $20 \%$ fetal bovine serum was added to the filter "spotted" and placed in a petri dish. Alternatively, PBS at pH 3.3, 7.4, or 10 were used as eluent buffer. The petri dish was then placed on a tilting plate (therefore, under stirring) for 30 minutes at room temperature. The residual volume of eluent (i.e., not absorbed by the filter) was recovered from the plate, and the contained viral titer was evaluated as described above. Moreover, in order to extract the elution buffer that remained absorbed in the filter at the end of the method, the procedure was integrated with a further step: after having recovered the residual volume of buffer, the filter was taken with sterile forceps, placed in a $50 \mathrm{ml}$ sterile syringe, and subjected to pressure, so as to release the volume of liquid still adsorbed in the filter. This was added to that previously collected from the plate and the viral titer contained therein was evaluated.

For the vortexing procedure, the spotted filter was transferred to a sterile $50 \mathrm{ml}$ tube and $4 \mathrm{ml}$ of DMEM supplemented with $2 \%, 10 \%$, or $20 \%$ fetal bovine serum was added. The tube was vortexed for 30 seconds at room temperature, and finally centrifuged at $1200 \mathrm{rpm}$ at room temperature for 10 minutes. The residual volume of eluent was then collected, and the viral titer was assessed as described above. 
For the shaking procedure, the spotted filter was transferred to a sterile 50ml tube and 8ml of DMEM supplemented with $10 \%, 20 \%$, or $40 \%$ fetal bovine serum was added. The test tube was placed on an orbital shaker and stirred for 60 minutes or 180 minutes at about 150 revolutions per minute. The residual volume of eluent was then collected and the viral titer contained was evaluated as described above. Alternatively, a combination of the shaking and vortexing procedure was tested, by vortexing the shaken sample for 30 seconds, then spinning it at $1200 \mathrm{rpm}$ at room temperature for 10 minutes and recovering the obtained supernatant.

For each single experiment, the viral inoculum itself was titrated and percentages of infective particles recovery were calculated according to the following formula: \% of recovery $=($ FFU recovered $x 100)$ / FFU inoculated.

Tests were also carried out to optimize storage times and temperatures (room temperature, $4^{\circ} \mathrm{C}$, $20^{\circ} \mathrm{C},-80^{\circ} \mathrm{C}$ ) of the spotted filters before they were subjected to elution techniques.

\section{PTFE filters}

A fixed inoculum of $\mathrm{HCoV}-\mathrm{OC} 43$ suspension $(50 \mu \mathrm{l})$ was spotted on PTFE filters of $4.7 \mathrm{~cm}$ diameter. The shaking procedure was selected to elute the viral particles entrapped in the filters, with some modifications: briefly, the spotted filter was transferred to a sterile $50 \mathrm{ml}$ tube and $8 \mathrm{ml}$ of DMEM supplemented with $10 \%$ fetal bovine serum was added. The test tube was placed on an orbital shaker and stirred for 60 minutes at about 150 revolutions per minute. The residual volume of eluent was then collected and the viral titer contained was evaluated as described above. For each single experiment, the viral inoculum itself was titrated and percentages of infective particles recovery were calculated according to the following formula: $\%$ of recovery $=(\mathrm{FFU}$ recovered $\mathrm{x} 100) / \mathrm{FFU}$ inoculated

Tests were also carried out to optimize storage times and temperatures (room temperature, $4^{\circ} \mathrm{C}$, $20^{\circ} \mathrm{C},-80^{\circ} \mathrm{C}$ ) of the spotted filters before they were subjected to elution techniques.

\section{Recovery tests with aspiration}

\section{Glass-fiber filters}

A fixed inoculum of $\mathrm{HCoV}-\mathrm{OC} 43$ suspension $(50 \mu \mathrm{l})$ was spotted on glass-fiber filters of $10 \mathrm{~cm}$ diameter and placed on a sampling head (the laboratory setup is showed in Figure 1). The spotted filters were aspirated for different times (namely, 1 minute, 5 minutes, 10 minutes, 15 minutes, and 20 minutes) at a flow rate of 500 liter of air per minute. The shaking procedure integrated with the 
vortexing step was selected to elute the viral particles entrapped in the filters. The residual volume of eluent was then collected and the viral titer contained was evaluated as described above.

\section{PTFE filters}

A fixed inoculum of $\mathrm{HCoV}-\mathrm{OC} 43$ suspension $(50 \mu \mathrm{l})$ was spotted on PTFE filters of $4.7 \mathrm{~cm}$ diameter and placed on a sampling head. The spotted filters were aspirated for different times (namely, 5 minutes, 15 minutes, 60 minutes, 180 minutes, and 900 minutes) at a flow rate of 20 liter of air per minute. The shaking procedure was selected to elute the viral particles entrapped in the filters, with some modifications: briefly, the spotted filter was transferred to a sterile $50 \mathrm{ml}$ tube and $8 \mathrm{ml}$ of DMEM supplemented with $10 \%$ fetal bovine serum was added. The test tube was placed on an orbital shaker and stirred for 60 minutes at about 150 revolutions per minute. The residual volume of eluent was then collected and the viral titer contained was evaluated as described above.

\section{Virus inactivation assays}

In order to evaluate the possible effect of the filter materials or of their components eventually released during the elution process, viral inactivation assays were performed. Briefly, both glass-fiber filters and PTFE filters were subjected to the shaking protocol or the combined shaking and vortexing protocol as described above. Subsequently, the residual volume of eluent was inoculated with approximately $10^{5} \mathrm{FFU}$ of $\mathrm{HCoV}-\mathrm{OC} 43$ and incubated at room temperature for 3 hours or 1 hour, respectively for glass-fiber filters and PTFE filters. Control samples were prepared by inoculating the same inoculum of fresh eluent. After the incubation, the samples were titrated and the residual viral infectivity was determined by indirect immunostaining as described above.

\section{SARS-CoV-2 Reverse Transcription-quantitative polymerase chain reaction (RT-qPCR)}

The samples are extracted in single with the MagMAX TM Viral/Pathogen Nucleic Acid Isolation Kit (ThermoFisher) protocol. $200 \mu \mathrm{l}$ of each sample were resuspended in $265 \mu 1$ of inactivating solution (binding solution), then the magnetic beads and proteinase $\mathrm{K}$ are added. The extraction procedure are carried out automatically using the King Fisher Flex instrumentation. At the end of the extraction process, the RNA extracted from the samples is resuspended in $50 \mu 1$ of elution solution.

The eluate obtained from the previous step are analyzed in duplicate by multiplex PCR using the SARS-CoV-2 ELITe MGB Kit (ElitechGroup). The targets are RdRp and ORF8 genes specific for SARS-CoV-2 and RNase P gene as endogenous Internal Control. The volume of sample loaded into 
PCR is $10 \mu \mathrm{l}$. The instrument used for PCR analysis is QuantStudio 5 Real-Time PCR (Applied Biosystems).

Data processing is carried out following the instructions of the PCR kit, setting the Thresholds for each individual gene and evaluating the presence of suitable PCR curves.

The results are expressed with the CT values (Cycle Threshold) for each detected target. In case of absence of amplification (absence of the desired target) the result is reported as Ct> 40. RT-qPCR assay limit of detection (LoD) can be conservatively defined as 5 genome copies per reaction, that is around 125 RNA copies per $\mathrm{mL}$ of transport medium.

The quantification curves have been determined by means of a SARS-CoV-2 RNA standard (LGC Standards): the RNA concentration of the transport medium (copies $/ \mathrm{mL}$ ) can be calculated, on average, as:

- $\quad 7 \cdot 10^{12} \cdot \operatorname{EXP}(-0.692 * \mathrm{CT})$ for ORF8 gene and

- $\quad 9 \cdot 10^{13} \cdot \operatorname{EXP}\left(-0.776^{*} \mathrm{CT}\right)$ for RdRp gene.

\section{HCoV-OC43 Reverse Transcription-quantitative polymerase chain reaction (RT-qPCR)}

The samples are extracted in single with the MagMAX TM Viral / Pathogen Nucleic Acid Isolation Kit (ThermoFisher) protocol. $200 \mu \mathrm{l}$ of each sample were resuspended in $265 \mu 1$ of the inactivating solution (binding solution), then the magnetic beads and proteinase $\mathrm{K}$ are added. The extraction procedure are carried out automatically using the King Fisher Flex instrumentation. At the end of the extraction process, the RNA extracted from the samples is resuspended in $50 \mu 1$ of elution solution.

The eluate obtained from the previous step is analyzed in single by multiplex PCR using the VIASURE Coronavirus 229E, NL63, OC43 \& HKU1 Real Time PCR Detection Kit (VIASURE). The target is a specific sequence for OC43. The volume of sample loaded into PCR is $5 \mu$. The CFX96 Touch Real-Time PCR (Bio-Rad) instrument was used for PCR analysis. Data processing was carried out by setting the Threshold above the non-specific background noise and evaluating the presence of suitable PCR curves.

\section{Sampling tests in real-life setting}

Based on the described sampling techniques and analytical methods, many field tests have been carried out involving indoor environments such as private houses, hospitals and means of transport. In particular we generally applied the biosampler device sampling $12.5 \mathrm{~L} / \mathrm{min}$ of air from 30 to 90 
minutes, a low volume pump sampling $20 \mathrm{~L} / \mathrm{min}$ on $4.7 \mathrm{~cm}$ PTFE filters for 60 minutes and a high volume device sampling $500 \mathrm{~L} / \mathrm{min}$ on $10 \mathrm{~cm}$ glass-fiber filters for 20 minutes. These sampling techniques have been applied both singularly and contemporarily.

After the sampling phase, filters are immersed in $10 \mathrm{~mL}$ DMEM and transported to the laboratory at a temperature about $4^{\circ} \mathrm{C}$. The transport normally takes less than two hours since the end of the sampling.

Once arrived at the laboratory, the transport medium is supplemented with a volume of FBS from 10 to $40 \%$ of the final volume. Samples are then subjected to the combined shaking-vortexing protocol as described above. The eluate $(13 \mathrm{~mL})$ is ultracentrifuged by using an ultracentrifuge Optima LE80K (Beckman Coulter Life Science) for 1 hour at 150000g; then, the supernatant is discarded, and the pelleted virus is concentrated in $0.3 \mathrm{~mL}$ of autoclave-sterilized PBS, and the presence of SARSCoV-2 genomic RNA is assessed by PCR.

Proof-of-concept experiments were performed to validate this approach by measuring the percentage of recovery and the capacity of ultracentrifugation to concentrate the eluted coronavirus. Briefly, 500000 FFU of HCoV-OC43 were inoculated in $13 \mathrm{~mL}$ of DMEM supplemented with 40\% FBS, then subjected to ultracentrifuge and titrated as previously described. For each single experiment, the viral inoculum itself was titrated and percentage of infective particles recovered after ultracentrifugation was calculated according to the following formula: \% of recovery $=(\mathrm{FFU}$ recovered x 100) / FFU inoculated.

\section{Statistical analysis}

Where possible descriptive statistic values (i.e. mean, median, and 95\% confidence interval (CI)) were calculated with GraphPad PRISM 7 software (GraphPad Software, San Diego, CA, USA). Where appropriate, regression analysis was performed to assess a correlation between $\mathrm{Ct}$ detected resulting from Real Time PCR and FFU/mL obtained by in vitro titration, using GraphPad PRISM 7. The square of the correlation coefficient $\left(\mathrm{R}^{2}\right)$ was used to value the linear regression. One-way ANOVA, followed by Bonferroni test, was used to assess the statistical significance of the differences between treated and untreated samples, where appropriate. Significance was set at the $95 \%$ level. 


\section{Results}

The results of the wash elution protocol on glass-fiber filters are presented in Figure 2A. DMEM supplemented with $2 \%$ of FBS was firstly chosen as the eluent since it is the medium canonically used to culture $\mathrm{HCoV}-\mathrm{OC} 43$ on MRC-5 cells. In this setting, the percentage of recovery of viral particles was very modest, about $2.7 \%$. Since the glass-fiber filters are highly absorbent, the protocol was integrated with a further step to recover the eluent soaked in the filter by pressing it inside a sterile syringe. The addition of this step in the procedure partially increased the percentage of virus recovered $(5.4 \%)$, yet not statistically significant ( $\mathrm{p}_{\text {ANOVA }}>0.05$ ). Extending the incubation time of the filter with the eluent up to 60 minutes resulted in a low recovery (1.6\%), probably due to a loss of viral titer due to excessive incubation of the virus at room temperature. As depicted, the use of PBS at different pHs as eluent buffer did not allow the recovery of infectious viral particles. By contrast, the percentage of recovered viral particles was increased to 12\%, when DMEM supplemented with $20 \%$ FBS was used as eluent buffer, a result significantly different if compared to the ones obtained with lower percentages of serum $\left(0.01<\mathrm{p}_{\mathrm{ANOVA}}<0.05\right)$ or $\mathrm{PBS}\left(\mathrm{p}_{\mathrm{ANOVA}}<0.001\right)$.

In order to evaluate the effect of a mechanical stimulus on the adhesiveness of viral particles trapped in the glass-fiber filters, two different approaches were tested: a first procedure based on vortexing the filter together with the eluent, and a second one based on shaking them on an orbital shaker. The results of the "vortex" protocol are depicted in figure 2B: while they are generally similar to the ones of the "wash" protocol, they clearly indicate that the increase of FBS in the eluent improves the release of the entrapped infective $\mathrm{HCoV}-\mathrm{OC} 43$ particles (ranging from $3.6 \%$ to $8.6 \%$, respectively for $\mathrm{DMEM}+10 \% \mathrm{FBS}$ and DMEM+20\% FBS). The shaking protocol resulted in even lower recovery efficiencies (Figure 2C), even increasing the percentage of FBS supplemented in the eluent (2.6\% of recovery and $1.8 \%$ of recovery respectively for DMEM+20\% FBS and DMEM+40\% FBS).

We therefore tested a combined protocol in which a shaking step of 60 minutes was followed by a vortexing step of 30 seconds. The results (depicted in Figure 3A) show a relevant improvement if compared with the one of the previously tested protocols, resulting in the recovery of HCoV-OC43 infective particles of $22.7 \%, 41.9 \%$, and $25.5 \%$, respectively for DMEM+10\% FBS, DMEM+20\% FBS, and DMEM+40\% FBS. A further improvement was obtained by increasing the shaking time of the glass-fiber filter together with the eluent to 180 minutes (eluent: DMEM+40\% FBS): according to the statistical analysis of the percentages obtained in each single independent experiment $(n=16$; figure $3 \mathrm{~B}$ ), an average percentage of recovery of $43.7 \%$ can be obtained with this protocol, with a 95\% CI between $29.8 \%$ and $57.6 \%$. Each sample eluted with this protocol, in addition to having been subjected to titration, was tested by means of PCR for the presence of the genetic material of HCoV- 
OC43; a linear regression analysis was performed in order to test the correlation between the two endpoints. As depicted in Figure 2C, the titer of eluted infectious $\mathrm{HCoV}-\mathrm{OC} 43$ particles correlates by direct proportionality $\left(\mathrm{R}^{2}=0.8\right)$ with the threshold cycle measured by real time PCR.

Once a reliable elution method had been developed for glass-fiber filters, we tested the effect of aspiration on the viral titer recovered from filters. This set of experiments was performed both on glass-fiber filters and PTFE filters. As depicted in Figure 4A, percentages of recovery from glassfiber filters range from a minimum of $4.7 \%$ to a maximum of $19.3 \%$, independently from the time of aspiration, thus excluding a virucidal effect on the population of particles trapped in the filter, for example due to dryness by way of the air flow passing through filters. According to the statistical analysis of the percentages obtained in each single independent experiment ( $n=18$; figure 4B), an average percentage of recovery of $12.9 \%$ can be obtained with this protocol, with a $95 \%$ CI between $10.4 \%$ and $15.4 \%$. Moreover, as shown in figure $4 \mathrm{C}$, real time PCR detected the presence of $\mathrm{HCoV}$ OC43 genome in each of the tested sample, showing a direct correlation of threshold cycles assessed and viral titers measured.

The shaking protocol, with appropriate modifications (60 minutes at 150 revolutions per minute), was used to elute viral particles also from PTFE filters; in this set of experiments the effect of longer aspiration times on entrapped infective particles was tested. As shown in Figure 5, the percentage of eluted $\mathrm{HCoV}-\mathrm{OC} 43$ particles is inversely related to the aspiration time, ranging from a maximum of $65.7 \%$ at 5 minutes of aspiration to a minimum of $20.4 \%$ at 900 minutes of aspiration.

We performed viral inactivation assays to exclude that the filters subjected to elution processes released into the eluent substances capable of inactivating the infectivity of the HCoV-OC43 particles. The results shown in figure 6 demonstrate that HCoV-OC43 does not lose infectivity when it is inoculated in a known volume of eluent, which was previously put in contact with the glass-fiber or PTFE filters and subsequently subjected to all the steps of the elution process.

Furthermore, we assessed the effect of the storage temperature of the filters immersed in the transport medium on the infectivity of HCoV-OC43. Briefly, we inoculated the glass-fiber filters or PTFE filters with $\mathrm{HCoV}-\mathrm{OC} 43$, then immersed them in the eluent and stored them for 24 hours at room temperature, $4^{\circ} \mathrm{C},-20^{\circ} \mathrm{C}$, or $-80^{\circ} \mathrm{C}$. As shown in Figure 7 , storing the glass-fiber filters (panel A) at room temperature significantly $\left(\mathrm{p}_{\mathrm{ANOVA}}<0.001\right.$ ) reduced $\mathrm{HCoV}-\mathrm{OC} 43$ infectivity to undetectable levels. On the contrary, the viral genome was still detectable in all samples, as assessed by PCR, regardless of the storage temperature. We obtained similar results with the PTFE filters (Figure 7B), although the decrease in viral titer in samples stored at room temperature was considerably lower and not significant ( $\left.\mathrm{p}_{\text {ANOVA }}>0.05\right)$. 
Finally, we evaluated whether the titer of the inoculum spotted on the glass-fiber filters or PTFE filters affected the recovery percentage. As shown in Figure 8A, the recovery percentage in glassfiber filters ranges between $18 \%$ and $21 \%$, depending on whether $3,000,000$ or $300,000 \mathrm{FFU}$ are spotted, it decreases considerably up to $0.5 \%$ if the inoculum is equal to $30,000 \mathrm{FFU}$, while it is equal to $0 \%$ for inocula equal to 3,000 or $300 \mathrm{FFU}$. We obtained similar results with PTFE filters (figure $8 \mathrm{~B})$, demonstrating that the percentage of recovery decreases with direct proportionality with respect to the viral inoculum, from a maximum of $25 \%$ (inoculum: $3,000,000 \mathrm{FFU}$ ) to a minimum of $14 \%$ (inoculum: 3,000 FFU); in the sample inoculated with $300 \mathrm{FFU}$ the recovery was equal to $0 \%$. In addition, the eluate samples were subjected to PCR and we demonstrated that the viral genetic material was detectable even in samples where the infecting units of the virus (i.e. the FFUs) were not detectable.

According to the proof-of-concept experiments performed with HCoV-OC43, the applied ultracentrifugation step can concentrate the eluted viral suspension to maxima of 15 times (data not shown), while the recovery efficiency of ultracentrifugation (calculated by comparing the number of total FFU diluted in the initial volume before ultracentrifugation and the number of FFU recovered in the pellet after ultracentrifugation) is 57\% (data not shown). These results are particularly promising if compared with recovery efficiencies reported by Ahmed et al. (2020b), who tested the efficiency of seven wastewater virus concentration methods (adsorption-extraction with three different pre-treatment options, centrifugal filter device methods with two different devices, polyethylene glycol (PEG 8000) precipitation, and ultracentrifugation) on murine hepatitis virus (MHV), finding mean recoveries ranging from 26.7 to $65.7 \%$.

As far as sampling tests are concerned, we reported in Table 1 the results of 20 samples taken in a private house where a positive person has been isolated for more than 3 weeks. The table highlights that in the first phase of the disease indoor concentration with poor ventilation conditions could be higher than 40 RNA copies $/ \mathrm{m}^{3}$ of air; three different sampling devices, namely a biosampler run for 90 minutes at $12.5 \mathrm{~L} / \mathrm{min}$, a PTFE sampler run for 60 minutes at $20 \mathrm{~L} / \mathrm{min}$ and a glass-fiber filter high volume sampler run for 20 minutes at $500 \mathrm{~L} / \mathrm{min}$, could give comparable results. 


\section{Discussion}

The aim of this study is to develop a specific and repeatable method for the detection of coronavirus infectious particles in the air, by exploiting the devices and techniques currently used for sampling air in the external environment as long as indoor. In particular, among the various devices that can be used to sample bioaerosol containing viruses, we focused on the use of filters because they appear to be the most effective device both to capture submicrometric particles and collect large air volumes at the same time.

The use of air filtration allows the recovery by aspiration of the viral particles present in the air, and their consequent trapping inside the filters, thus requiring a subsequent elution step to extract the virus from the solid matrix in which it is sequestered. For this purpose, we evaluated different types of eluents; based on our data, the addition of FBS to the elution buffer is essential to trigger a more conspicuous release of viral particles. We showed that HCoV-OC43 infective particles can be recovered from both PTFE and glass-fiber filters, and that these materials do not inactivate viral particles. We identified DMEM supplemented with 40\% FBS as the best eluent for glass-fiber filters, and DMEM supplemented with 10\% FBS as the most suitable one for PTFE filters.

While PTFE filters grant the highest recovery percentages with the less complex procedures, glassfiber filters are more suitable for sampling procedures in a real-life setting, because they enable the recovery of a higher volume of air in the same time unit if compared to PTFE filters: for example, the use of a high volume sampler filtering on glass-fiber filters allows the sampling of 10 cubic meter of air in 20 minutes, whereas one hour of sampling with PTFE filters collects 1 cubic meter of air.

For glass-fiber filters, we found that a two-step procedure is necessary to elute viral infective particles: a 3 hour-shaking step, followed by a 30 seconds-shaking step. For PTFE filters, a 60 minutes shaking procedure is adequate.

We have shown that a simple mechanical stimulus (pressure or agitation) is not sufficient to release the viral particles trapped in the glass-fiber filters but that a specific combination of mechanical stimuli with a suitable eluent is required to mediate their release. While this limits the amount of $\mathrm{HCoV}-\mathrm{OC} 43$ particles that can be recovered, on the other hand it demonstrates that they show a tenacious adhesiveness towards fiberglass, which makes these filters particularly suitable for capturing and trapping infectious particles sampled from the air.

Moreover, we demonstrated that the use of filters to capture bioaerosol is very efficient and, as far as the sampling duration is lower than 60 minutes, the expected inactivation of coronavirus is not drastic. Table 1 reports SARS-CoV-2 air concentration trends inside a private house, along with the development of the disease of the owner (more or less, 3 weeks, from the first positive test). These 
data highlight a clear decreasing trend of air concentration since the first days of the disease, that is to say that airborne virus tends to reflect viral load trends of patients. At the same time, it is possible to hypothesize that sampling on glass-fiber filters at very high flow rates make analytical sensitivity pretty higher than other sampling techniques.

Ii is important to underline that PTFE filters are made up of a porous membrane with a complex structure with tortuous routes through the filter material, while glass-fiber filters are fibrous filters consisting of a deep mesh of fibers with random orientations.

As a matter of facts, aerosol filtration is far more complex than a simple "sieve" model as it is based on 5 different mechanism, namely interception, impaction, diffusion (Brownian motion), electrostatic attraction, and sedimentation. Therefore, aerosol filters can efficiently collect particles much smaller than would be expected on the basis of the pore size of the filter.

PTFE filters could reach very high collection efficiency, but they are generally characterized by high pressure drops (low flow rates can be realized). On the contrary, the high porosity of glass-fiber filters allows very high flow rates to be aspired and good collection efficiencies at the same time.

It should be remembered that recovery efficiency of the genetic materials coming from collected SARS-CoV-2 particles would be surely higher that the reported data on infectious virus.

\section{Real-life sampling test results}

On the basis of the sampling techniques and the analytical methods developed as described in the previous chapters, many field tests have been carried out involving COVID-19 hospital wards, private houses of infected families, outdoor air in the center of a 1 million people city.

The results obtained with a high level of confidence support the following considerations:

- in external environment, away from aggregated people, queues or crowded and narrow street, SARS-CoV-2 has not been detectable in air;

- in hospital rooms or spaces, and in particular in wards with the presence of patients with high viral loads, the detectable concentrations of SARS-CoV-2 were generally very low, lower than 10 genomic copies per cubic meter, also because of the high air ventilation rates (6-12 air changes per hour);

- in domestic environment, on the contrary, virus concentrations have been found to be more consistent, up to $40 \div 50$ genomic copies of the virus per cubic meter of air. These values are strongly influenced by the air changes frequencies and the number of positive subjects present 
in the house, as well as by the development of the most common symptoms of the disease (dry cough).

The results described confirm those reported by some studies published by Liu et al. (2020) and Stern et al. (2021), reporting concentrations in the range $5 \div 50$ copies $/ \mathrm{m} 3$ of air within hospital wards, even though these results were not supported by the use of validated analytical methods such as those described in the present paper.

The described sampling and analytical methods are very interesting in order to compare different sampling techniques as well, for example different filtering materials (PTFE vs fiber glass) and different capture mechanisms (filtering vs impaction), by carrying out air sampling with different devices in the same room, simultaneously.

\section{Conclusions}

In the present paper we described the development of reliable sampling and pretreatment methods in order to quantify the presence of SARS-CoV-2 in air as bioaerosol.

The study defines in particular samples pretreatment aiming at optimizing virus recovery from glassfiber and PTFE filters. Recovery test results focused on the sample concentration step carried out by means of ultracentrifugation are also reported.

On the basis of the described approach, we can conclude that:

- the recovery efficiency of HCoV-OC43 from PTFE filters is much higher and easier than from glass-fiber filters or quartz filter; for glass-fiber filters, we found that a two-step procedure is necessary to elute viral infective particles: a 3 hour-shaking step, followed by a 30 secondsshaking step. For PTFE 60 minutes-shaking is enough.

- the recovery efficiency from glass-fiber filters and quartz filters could be strongly enhanced by using an elution buffer containing up to $40 \%$ of fetal calf serum;

- the effect of suction time on filters could be resumed as follows: concerning $10 \mathrm{~cm}$ glass-fiber filters, sampling durations up to 20 minutes at a flow rate of $500 \mathrm{~L}$ per minute do not affect recovery efficiencies. On the contrary, the recovery efficiency of infectious virions from $4.7 \mathrm{~cm}$ PTFE filters decreases of a factor 2 after 3 hours of sampling at a flow rate of $20 \mathrm{~L}$ per minute;

- the applied ultracentrifugation step can concentrate the eluted viral suspension to maxima of 15 times, while the recovery efficiency of ultracentrifugation is $57 \%$. 
The developed methods, aiming at providing the community with reliable determinations about the presence of SARS-CoV-2 and other airborne pathogens in air, proves essential for the development, during the pandemic, of a coherent management of places, in particular crowded ones such as means of transport, stations, gyms, theaters, cinemas. In this regard, the studies described here provide a solid basis for developing reliable methods for quantifying the viral load in the air, allowing, in perspective, to assess the degree of health risk in a specific environment. They also represent an important acquisition of technical knowledge that can be readily applied in the future in the case of the emergence of new epidemics or pandemics.

\section{Declaration of interest}

The authors declare that they have no known competing financial interests or personal relationships that could have appeared to influence the work reported in this paper.

\section{Acknowledgements}

The authors acknowledge the financial support received from Assicurazioni Generali and Intesa San Paolo to realize the Environmental Virology laboratory run by Arpa Piemonte (Environmental Protection Agency of Piedmont) in La Loggia, Torino, Italy. The authors are grateful to the Molecular Virology Laboratory at Dept. of Clinical and Biological Science, Università degli Studi di Torino, for the technical support received. 


\section{References}

Ahmed, W., Bertsch, P., Bivins, A., Bibby, K., Farkas, K., Gathercole, A., et al., 2020b. Comparison of virus concentration methods for the RT-qPCR-based recovery of murine hepatitis virus, a surrogate for SARS-CoV-2 from untreated wastewater. Science of the Total Environment 739 (2020) 139960.

ASHRAE Position Document on Infectious Aerosols Approved by ASHRAE Board of Directors April 14, 2020.

ASHRAE EPIDEMIC TASK FORCE, 2021. Core Recommendations for Reducing Airborne Infectious Aerosol Exposure.

Atkinson, J., Chartier, Y., Lúcia Pessoa-Silva, C., Jensen, P., Li, Y., Seto, W.-H., 2009. Natural Ventilation for Infection Control in Health-Care Settings. WHO, Geneva.

Da Silva, P.G., Nascimento, M.S.J., Soares, R.R.G., Sousa, S.I.V., Mesquita, J.R., 2021. Airborne spread of infectious SARS-CoV-2: Moving forward using lessons from SARS-CoV and MERS-CoV. Sci Total Environ. 2021 Apr 10;764:142802.

Platto, S, Xue. T,, Carafoli. E., 2020. COVID19: an announced pandemic. Cell Death Dis. 2020 Sep 24;11(9):799.

Binder, R.A., Alarja, N.A., Robie, E.R., Kochek, K.E., Xiu, L., Rocha-Melogno, L., Abdelgadir, A., Goli, S.V., Farrell, A.S., Coleman, K.K., Turner, A.L., Lautredou, C.C., Lednicky, J.A., Lee M.J., Polage, C.R., Simmons, R.A., Deshusses, M.A., Anderson, B.D., Gray, G.C., 2020. Environmental and Aerosolized Severe Acute Respiratory Syndrome Coronavirus 2 Among Hospitalized Coronavirus Disease 2019 Patients. J Infect Dis. 2020 Nov 9;222(11):1798-1806.

CDC (Center for Disease Control and Prevention, USA), 2021. Science Brief: SARS-CoV-2 and Surface (Fomite) Transmission for Indoor Community Environments.

Lednicky, J.A., Lauzardo, M., Fan, Z.H., Jutla, A., Tilly, T.B., Gangwar, M., Usmani, M., Shankar, S.N., Mohamed, K., Eiguren-Fernandez, A., Stephenson, C.J., Alam, M.M., Elbadry, M.A., Loeb, J.C., Subramaniam, K., Waltzek, T.B., Cherabuddi, K., Morris, J.G. Jr, Wu, C.Y., 2020. Viable SARS-CoV-2 in the air of a hospital room with COVID-19 patients. Int J Infect Dis. 2020 Nov;100:476-482.

Lin, X., Reponene, T.A., Willeke, K., Grinshpun, S.A., Foarde, K.K., Ensor, D.S., 1999. Long-term sampling of airbome bacteria and fungi into a non-evaporating liquid. Atmospheric Environment 33 (1999) 4291-4298. 
Liu, Y., Ning, Z., Chen, Y., Guo, M., Liu, Y., Gali, N.K,, et al. 2020. Aerodynamic analysis of SARSCoV-2 in two Wuhan hospitals. BioRxiv.

Morawska, L., Cao, J., 2020. Airborne transmission of SARS-CoV-2: the world should face the reality. Environ. Int. 139, 105730.

Pan, M., Lednicky, J.A., Wu, C.-Y., 2019. Collection, particle sizing and detection of airborne viruses. Journal of Applied Microbiology ISSN 1364-5072.

Robotto, A., Quaglino, P, Lembo, D., Morello, M., Brizio, E. Bardi, L., Civra, A., 2021. SARS-CoV2 and indoor/outdoor air samples: a methodological approach to have consistent and comparable results. Environmental Research 195 (2021) 110847

Santarpia,J.L., Rivera, D.N., Herrera, V.L., Morwitzer, M.J., Creager, H.M., Santarpia, G.W., Crown, K.K., Brett-Major, D.M., Schnaubelt, E.R., Broadhurst, M.J., Lawler, J.V., Reid, S.P., Lowe, J.J., 2020. Aerosol and surface contamination of SARS-CoV-2 observed in quarantine and isolation care. Sci Rep. 2020 Jul 29;10(1):12732.

Scherwing, C., Bunke, J., 2019. Continuous Microbial Air Monitoring in Clean Room Environments. Sartorius application note.

Siegel, J.D., Rhinehart, E., Jackson, M., Chiarello, L., 2007. 2007 guideline for isolation precautions: preventing transmission of infectious agents in healthcare settings (updated July 2019). Centers Dis. Control Prev 1-232.

Stern, R.A., Koutrakis, P., Martins, M.A.G., Lemos, B., Dowd, S.E., Sunderland, E.M., Garshick, E., 2021. Characterization of hospital airborne SARS-CoV-2. Respir Res (2021) 22:73 https://doi.org/10.1186/s12931-021-01637-8

van Doremalen, N., Bushmaker, T., Morris, D.H., Holbrook, M.G., Gamble, A., Williamson, B.N., Tamin, A., Harcourt, J.L., Thornburg, N.J., Gerber, S.I., Lloyd-Smith, J.O., de Wit, E., Munster, V.J., 2020. Aerosol and Surface Stability of SARS-CoV-2 as Compared with SARS-CoV-1. N Engl J Med. 2020 Apr 16;382(16):1564-1567.

Verreault, D., Moineau, S. and Duchaine, C., 2008. Methods for sampling of airborne viruses. Microbiol Mol Biol R 72, 413-444.

WHO, 2020c. Transmission of SARS-CoV-2 - Implications for Infection Prevention Precautions: Scientific Brief [WWW Document]. World Health Organization URL. https://www.who.int/news-room/commentaries/detail/transmission-of-sars-cov-2implications-for-infection-prevention-precautions. 
Willeke, K., Lin, X., Grinshpun, S.A., 1998. Improved aerosol collection by combined impaction and centrifugal motion. AEROSOL SCIENCE AND TECNOLOGY 28:439-456 (1998) (c) 1998 American Association for Aerosol Research.

Zhang, R., Li, Y., Zhang, A.L., Wang, Y., Molina, M.J., 2020. Identifying airborne transmission as the dominant route for the spread of COVID-19. Proc Natl Acad Sci U S A. 2020 Jun 30;117(26):14857-14863. 


\section{Legends}

Figure 1. Experimental setting for recovery tests with aspiration in biosafety level 2 (BSL-2) conditions. A fixed inoculum of HCoV-OC43 suspension $(50 \mu \mathrm{l})$ was spotted on glass-fiber filters of $10 \mathrm{~cm}$ diameter and placed on a sampling head (A), and a high-volume sampler for air filtration was used (B).

Figure 2. Evaluation of the extraction efficiency of infective $\mathrm{HCoV}-\mathrm{OC} 43$ particles from $4.7 \mathrm{~cm}$ glass-fiber filters, accordingly to the wash procedure (panel A), vortexing procedure (panel B), or the shaking procedure (C). The titers of filter-recovered HCoV-OC43 are expressed as a percentage of the titer of the inoculated virus (\% of recovery $=($ FFU recovered $x 100) / F F U$ inoculated $)$. Error bars represent the standard error of the mean (SEM) of independent experiments. ${ }^{*} \mathrm{p}_{\mathrm{ANOVA}}<0.05$; $* * p_{\text {ANOVA }}<0.01 ; * * *$ p $_{\text {ANOVA }}<0.001$

Figure 3. Evaluation of the extraction efficiency of infective $\mathrm{HCoV}-\mathrm{OC} 43$ particles from from 4.7 $\mathrm{cm}$ glass-fiber filters when using a combined shaking and vortexing procedure. In panel $\mathrm{A}$, the titers of filter-recovered $\mathrm{HCoV}-\mathrm{OC} 43$ are expressed as a percentage of the titer of the inoculated virus (\% of recovery $=($ FFU recovered $x$ 100) $/$ FFU inoculated $)$. Error bars represent the SEM of independent experiments. In panel $\mathrm{B}$, the dispersion of the different percentages of recovery obtained with the shaking-vortexing and using DMEM+40\%FBS are depicted; the horizontal line represents the mean value of 16 independent experiments, while the error bars represent the SEM of independent experiments. In panel $\mathrm{C}$, a linear regression analysis correlates for each eluate obtained with the combined shaking and vortexing procedure (eluent: DMEM+40\%FBS), the FFU recovered with the respective threshold cycles $(\mathrm{Ct})$ measured with the quantitative real-time RT-PCR. 95\% CI: 95\% confidence interval; $\mathrm{R}^{2}=$ square of the correlation coefficient

Figure 4. Evaluation of the extraction efficiency of infective $\mathrm{HCoV}-\mathrm{OC} 43$ particles from $10 \mathrm{~cm}$ (panel A) glass-fiber filters when using a combined shaking and vortexing procedure. In both panels, the titers of filter-recovered $\mathrm{HCoV}-\mathrm{OC} 43$ are expressed as a percentage of the titer of the inoculated virus $(\%$ of recovery $=($ FFU recovered $x 100) /$ FFU inoculated $)$, and error bars represent the SEM of independent experiments. In panel $\mathrm{B}$, the dispersion of the different percentages of recovery obtained with the shaking-vortexing and using DMEM+40\%FBS (10 cm glass-fiber filters) are depicted; the horizontal line represents the mean value of 16 independent experiments, while the error 
bars represent the SEM of independent experiments. In panel C, a linear regression analysis correlates for each eluate obtained with the combined shaking and vortexing procedure $(10 \mathrm{~cm}$ glass-fiber filter; eluent: DMEM+40\%FBS), the FFU recovered with the respective threshold cycles $(\mathrm{Ct})$ measured with the RT-PCR. 95\% CI: 95\% confidence interval; $\mathrm{R}^{2}=$ square of the correlation coefficient

Figure 5. Evaluation of the extraction efficiency of infective $\mathrm{HCoV}-\mathrm{OC} 43$ particles from $4.7 \mathrm{~cm}$ PTFE filters when using the shaking procedure. The titers of filter-recovered HCoV-OC43 are expressed as a percentage of the titer of the inoculated virus $(\%$ of recovery $=($ FFU recovered $x 100)$ / FFU inoculated), and error bars represent the SEM of independent experiments. ${ }^{*} p_{\text {ANOVA }}<0.05$

Figure 6. Virus inactivation assay. The white bars refer to eluent samples inoculated with $\mathrm{HCoV}$ OC43 and previously exposed to PTFE or glass-fiber filters and subjected to the shake-vortex procedure. Control samples (black bars) were prepared by inoculating the same inoculum of fresh eluent. On Y axis, the titers of $\mathrm{HCoV}-\mathrm{OC} 43$ are expressed as FFU/ml. Error bars represent the SEM of independent experiments.

Figure 7. Effect of filter storage temperature on the extraction efficiency of infective HCoV-OC43 particles from $4.7 \mathrm{~cm}$ glass-fiber (A) or PTFE (B) filters. On Y axis, the titers of eluted HCoV-OC43 are expressed as total extracted FFU when using the combined shaking-vortexing procedure, and error bars represent the SEM of independent experiments. At the top of each bar, threshold cycle (Ct) values measured with RT-PCR are indicated for each sample.

Figure 8. Evaluation of the extraction efficiency of infective $\mathrm{HCoV}-\mathrm{OC} 43$ particles from $4.7 \mathrm{~cm}$ glass-fiber (A) or PTFE filters (B) as a function of viral inoculum. . On Y axis, the titers of infective $\mathrm{HCoV}-\mathrm{OC} 43$ inoculated on filters are expressed as total FFU. The titers of filter-recovered HCoVOC43 are expressed as a percentage of the titer of the inoculated virus $(\%$ of recovery $=(\mathrm{FFU}$ recovered x 100) / FFU inoculated), and error bars represent the SEM of independent experiments. At the top of each bar, threshold cycle $(\mathrm{Ct})$ values measured with RT-PCR are indicated for each sample. 

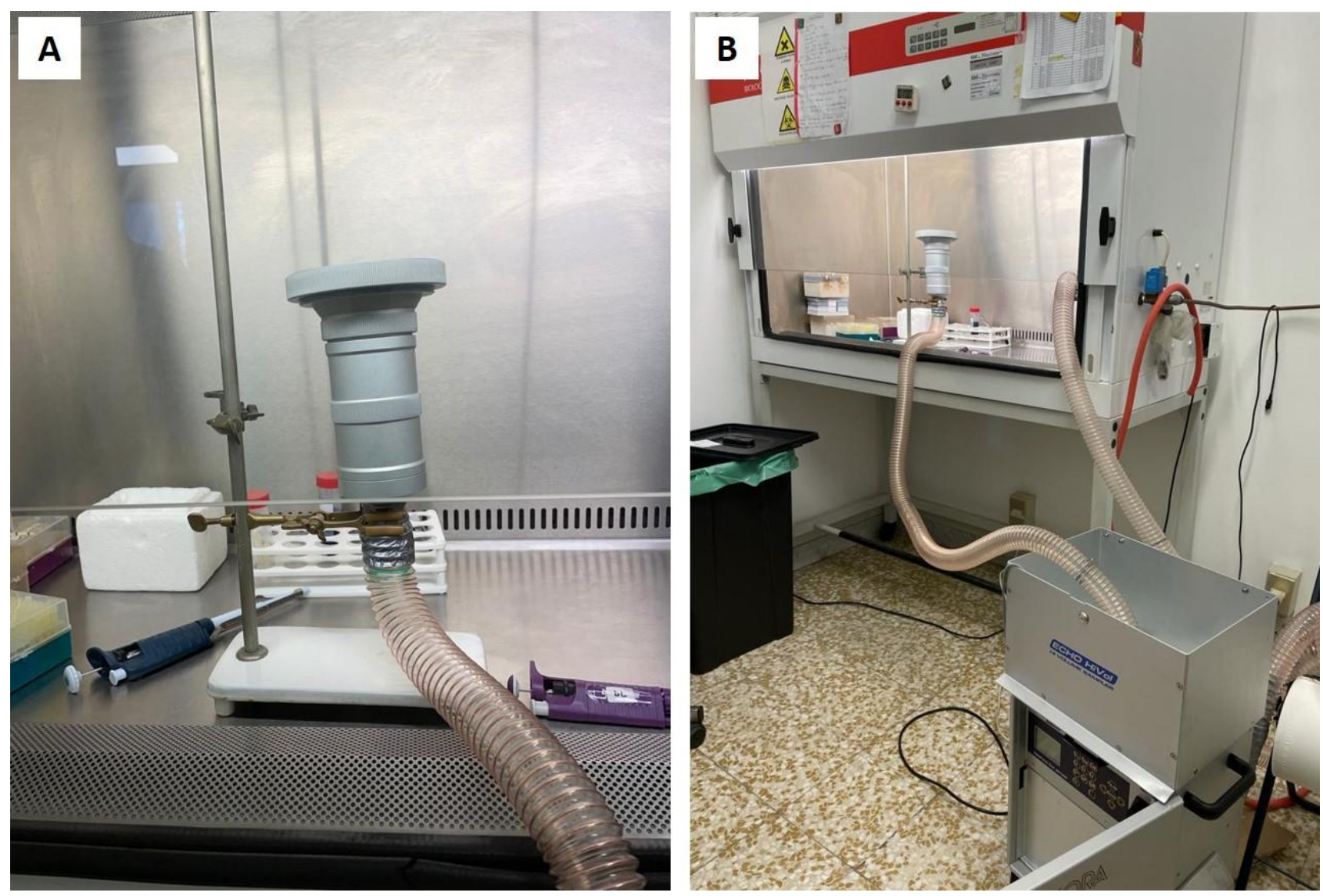

Figure 1. 

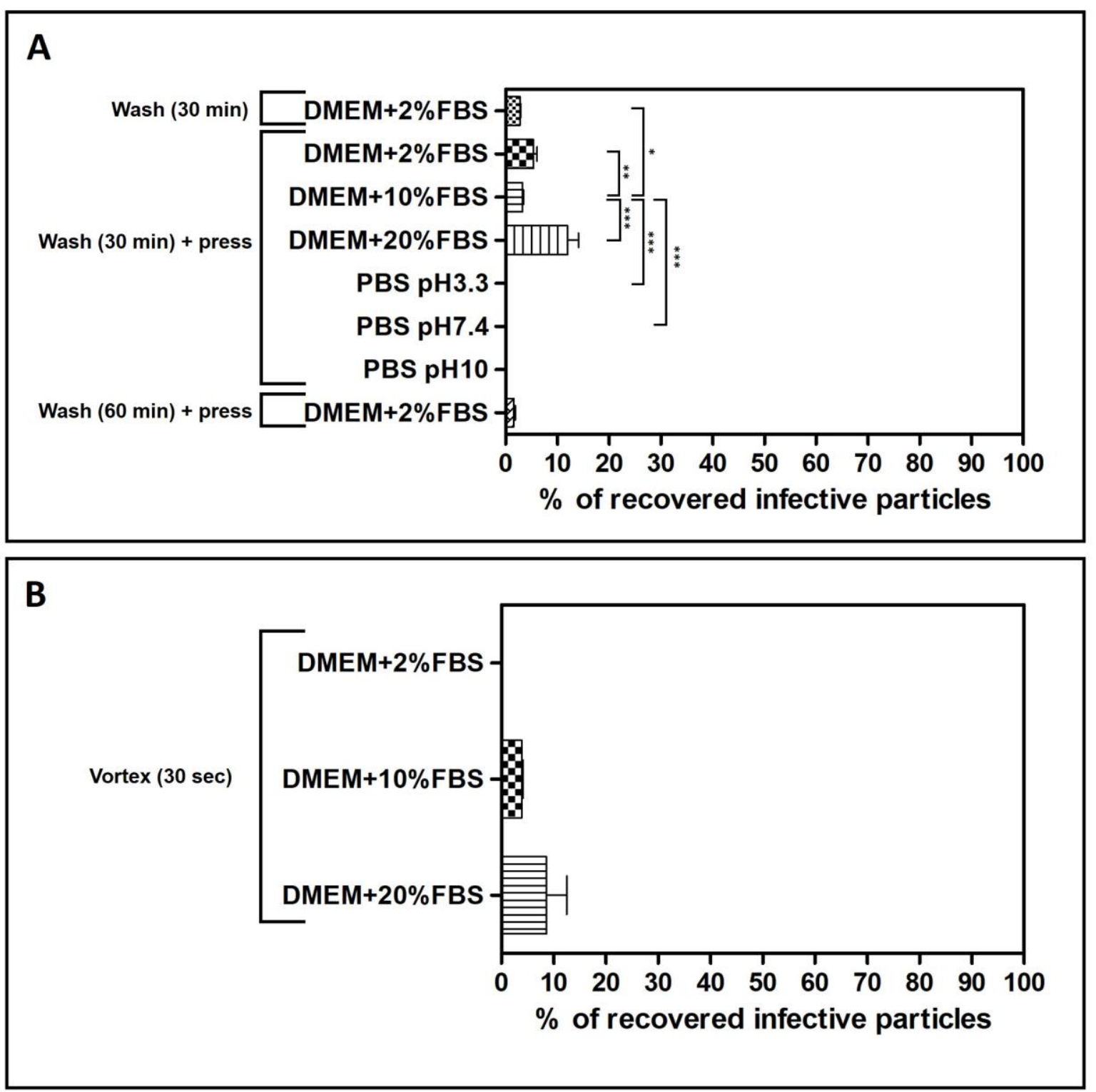

C

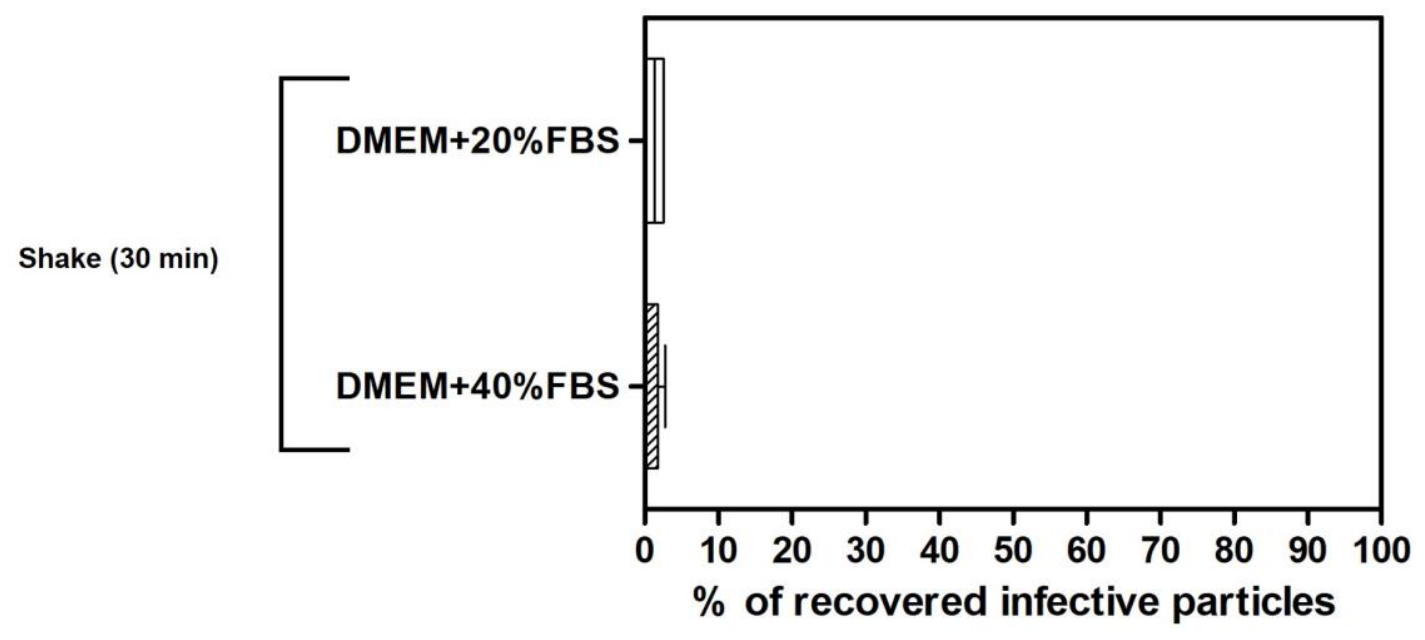

Figure 2. 

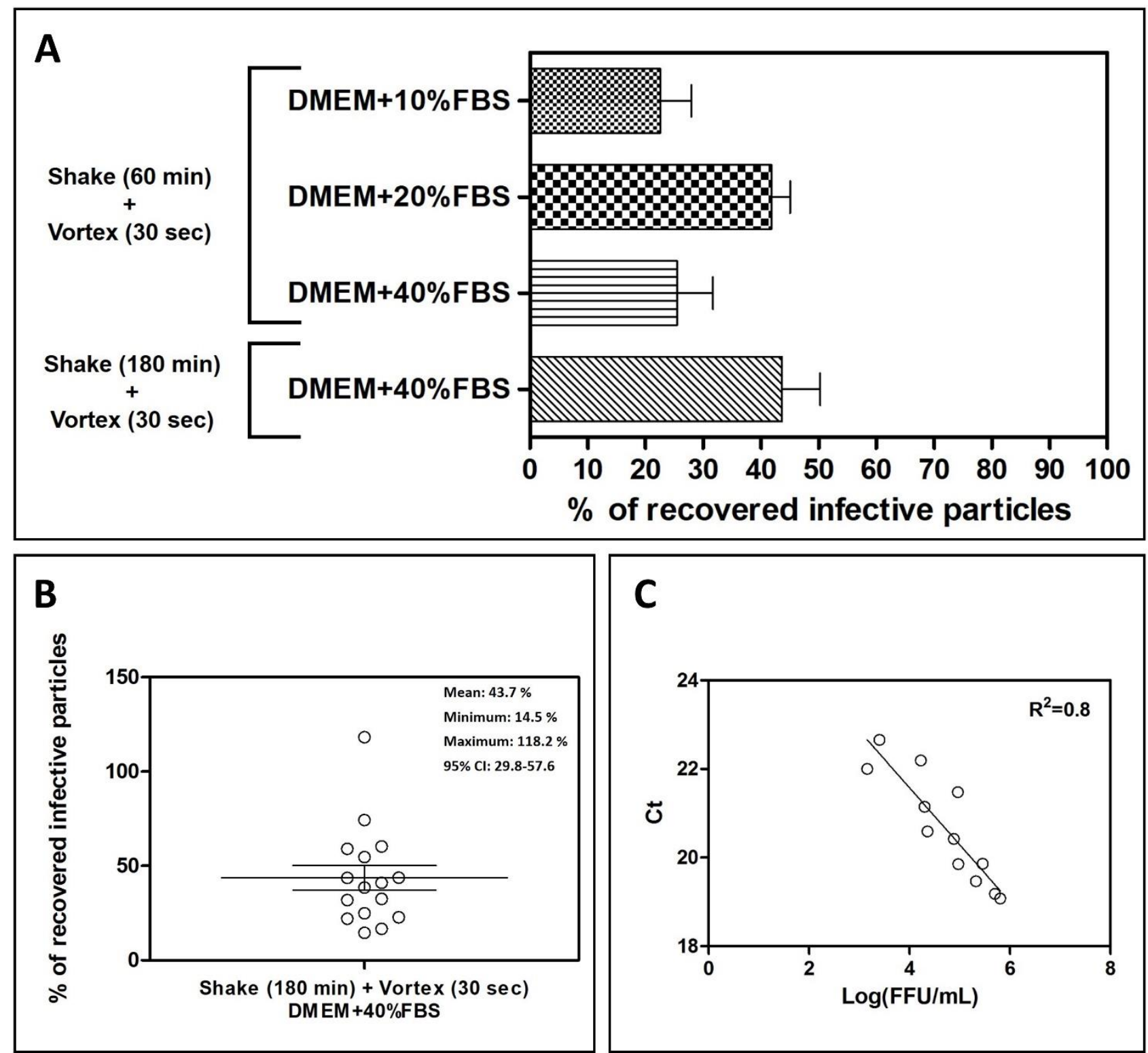

C

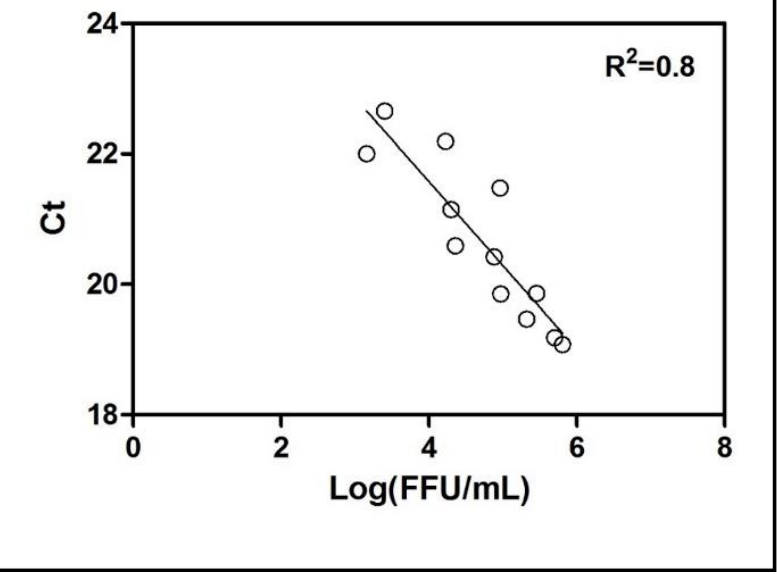

Figure 3. 

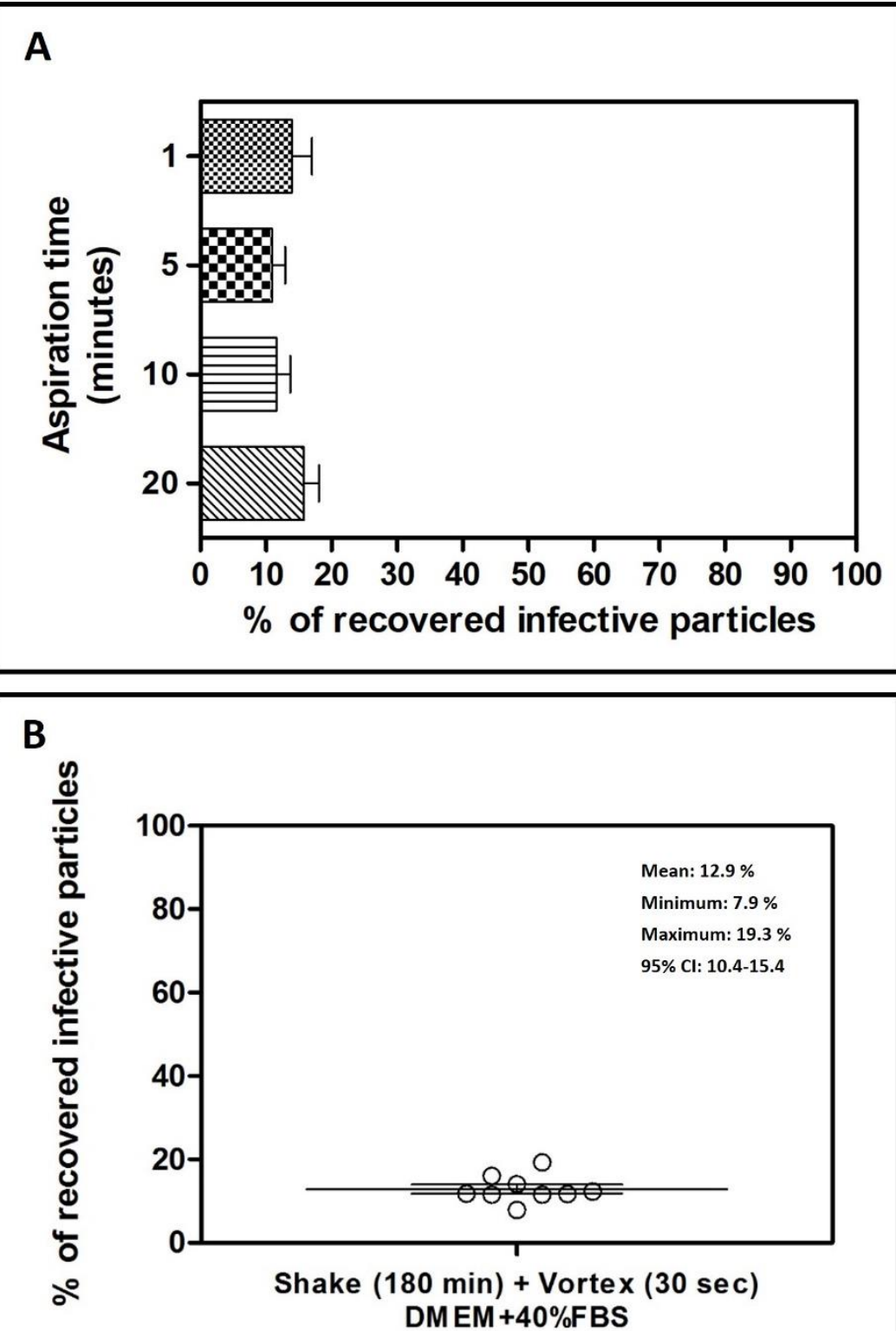

C

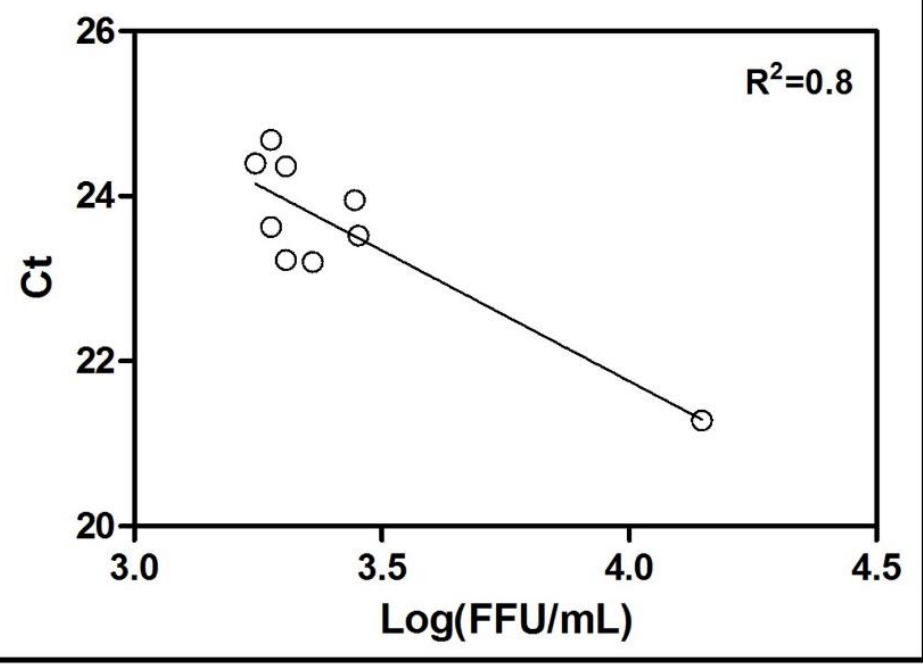

Figure 4. 


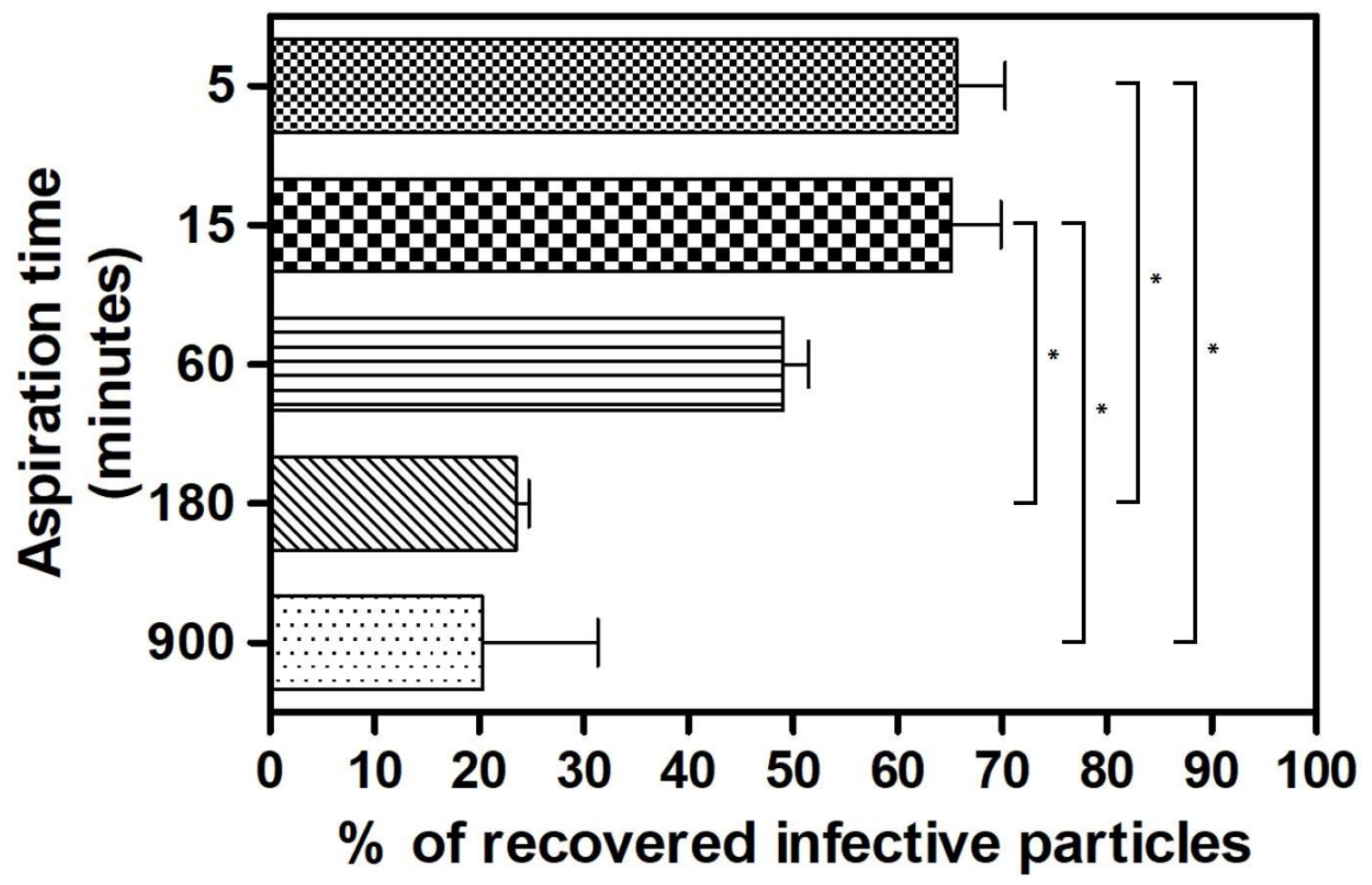

Figure 5. 
- Control eluent

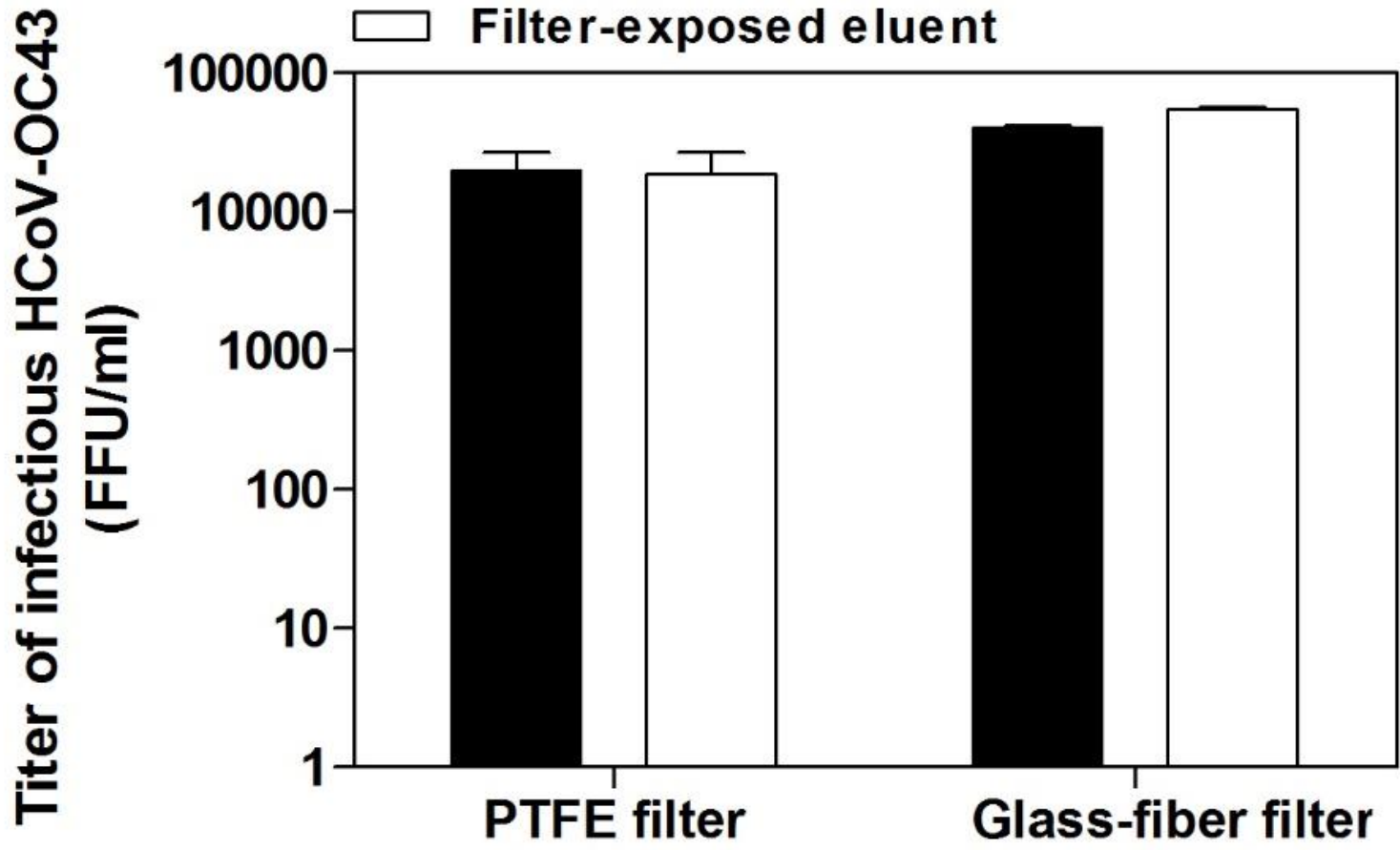

Figure 6. 


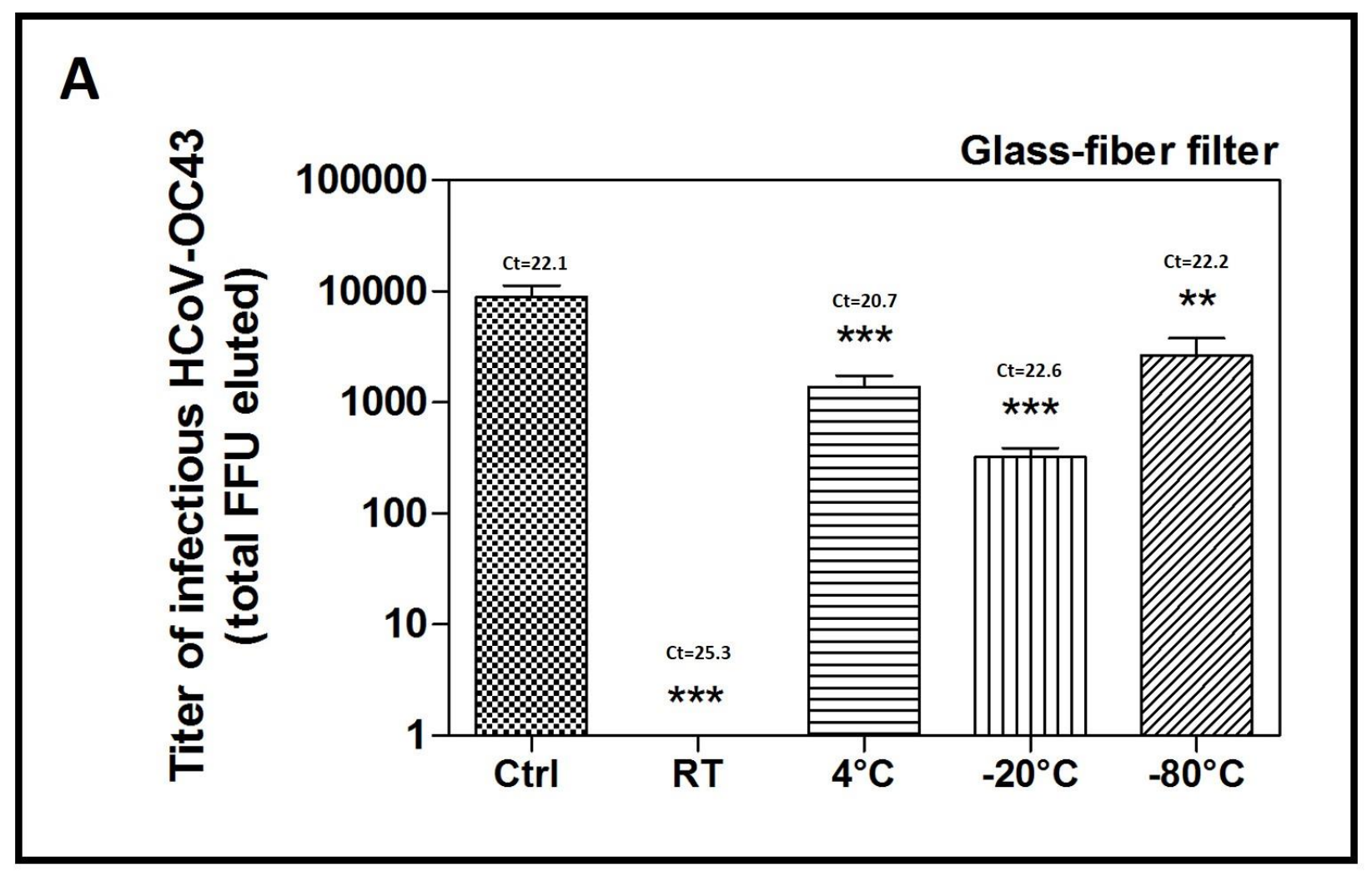

B

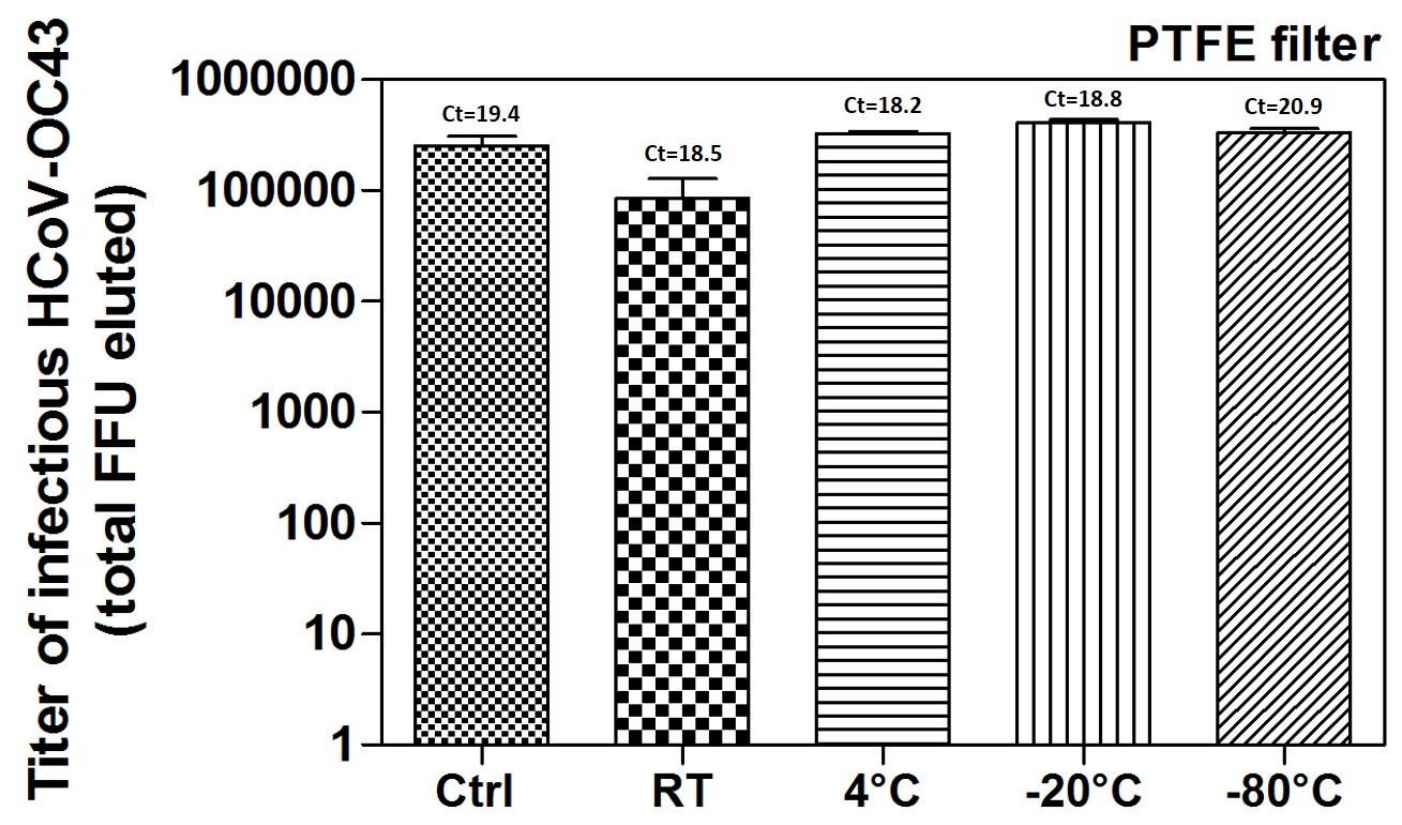

Figure 7. 
A

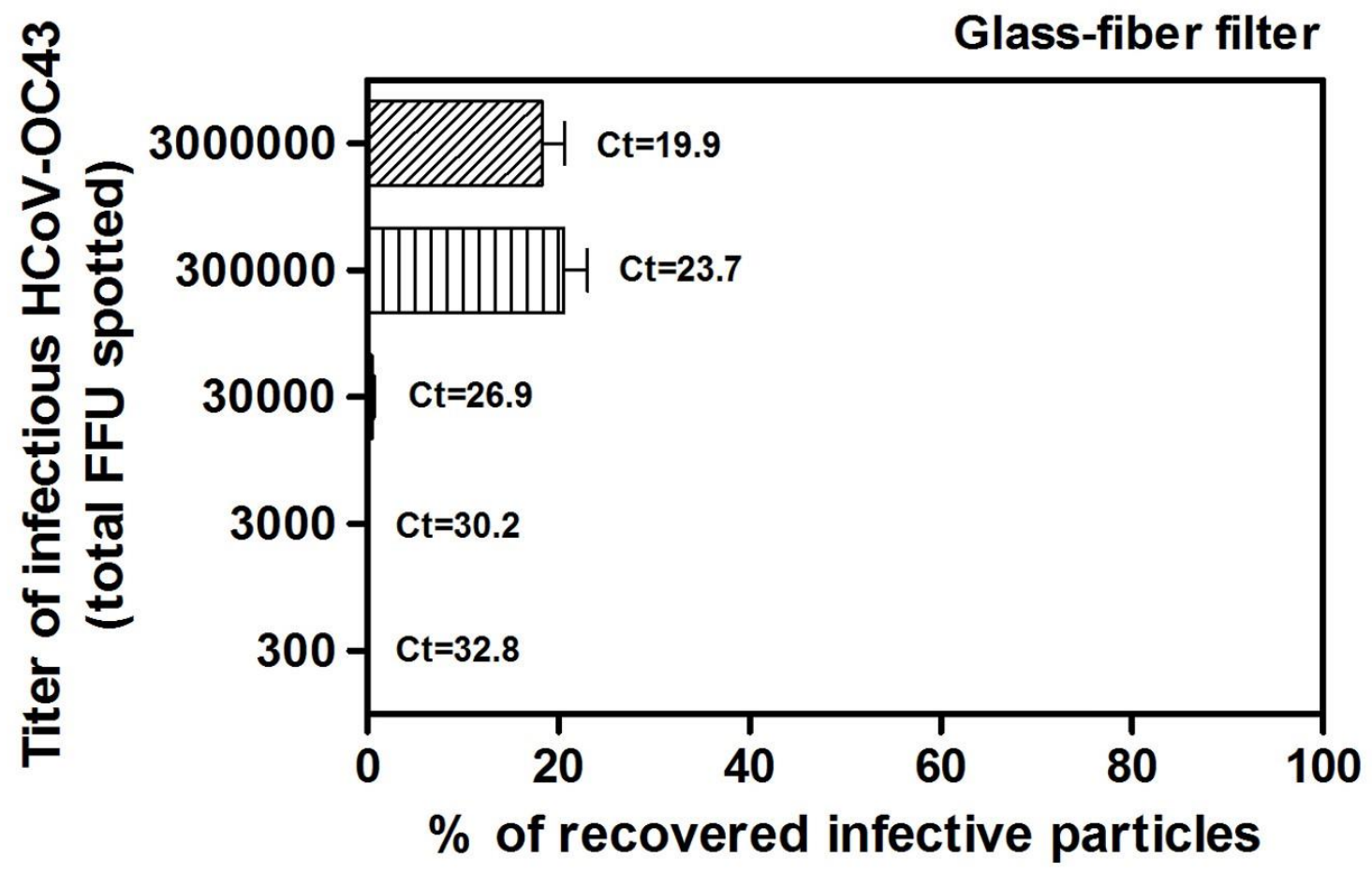

B

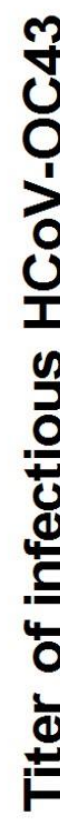

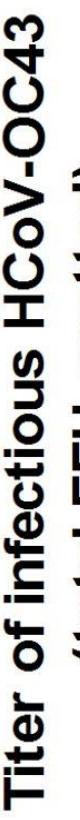

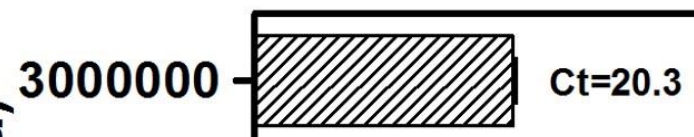

PTFE filter

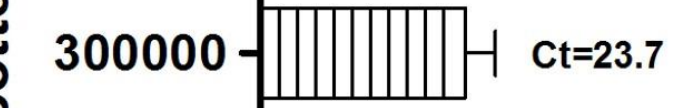

30000 尼

离

正
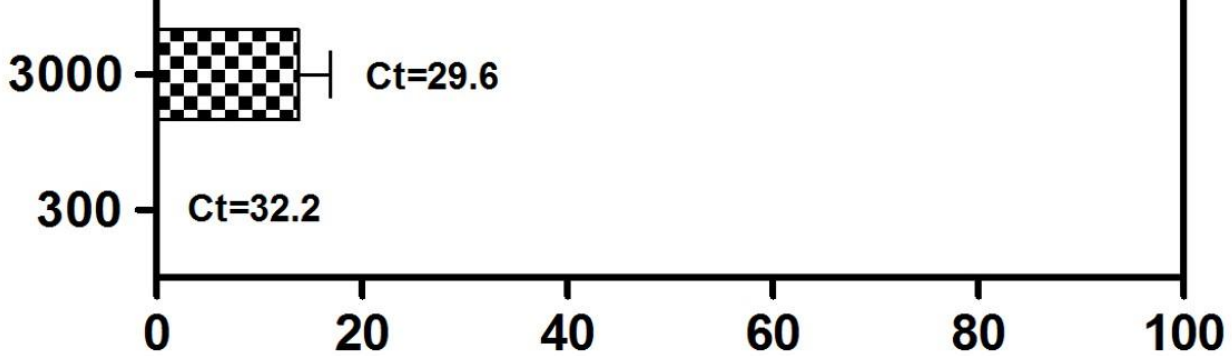

$\%$ of recovered infective particles

Figure 8. 
Table 1. SARS-CoV-2 air concentration, expressed as RNA copies per $\mathrm{m}^{3}$ of air, measured in a private house where a positive person has been isolated.

\begin{tabular}{|c|c|c|c|c|}
\hline \multirow{3}{*}{$\begin{array}{l}\text { days from } \\
\text { the 1st test }\end{array}$} & \multicolumn{3}{|c|}{ sampling methods (sampling duration, air volume sampled) } & \multirow{3}{*}{$\begin{array}{c}\text { sampling place (room, volume, } \mathrm{n}^{\circ} \\
\text { of patients) }\end{array}$} \\
\hline & PTFE (60min, $1 \mathrm{~m}^{3}$ ) & glass fiber $\left(20 \mathrm{~min}, 10 \mathrm{~m}^{3}\right)$ & biosampler (90 $\left.\min , 1.1 \mathrm{~m}^{3}\right)$ & \\
\hline & $\begin{array}{c}\text { copies } / \mathrm{m} 3, \text { mean values } \\
\text { ( } \mathrm{n}^{\circ} \text { of samples) }\end{array}$ & $\begin{array}{c}\text { copies/m3, mean values } \\
\text { ( } n^{\circ} \text { of samples) }\end{array}$ & $\begin{array}{c}\text { copies } / \mathrm{m} 3, \text { mean values }\left(\mathrm{n}^{\circ}\right. \\
\text { of samples) }\end{array}$ & \\
\hline 3 & $31.1(3)$ & $42.1(1)$ & - & office, $33 \mathrm{~m} 3,1$ patient inside \\
\hline 6 & - & - & $39.0(1)$ & bedroom, $55 \mathrm{~m} 3,1$ patient inside \\
\hline 20 & not detectable (1) & $8.1(2)$ & not detectable (1) & bedroom, $55 \mathrm{~m} 3,1$ patient inside \\
\hline
\end{tabular}

